\title{
Association between Germline Mutations in the RET Proto-Oncogene and Colorectal Cancer and Polyps
}

\author{
Rosner Guy ${ }^{1,2 *}$, Reicher Rel ${ }^{1}$, Carmi Shai ${ }^{3}$, Gluck Nathan ${ }^{1,2}$ and Kariv Revital ${ }^{1,2}$ \\ ${ }^{1}$ Department of Gastroenterology and Liver Diseases, Tel-Aviv Sourasky Medical Center, Israel \\ ${ }^{2}$ Sackler School of Medicine, Tel-Aviv University, Israel \\ ${ }^{3}$ Braun School of Public Health and Community Medicine, The Hebrew University of Jerusalem, Israel
}

Submission: December 12, 2018; Published: February 04, 2019

"Corresponding author: Guy rosner; Department of gastroenterology and liver diseases; Tel-aviv sourasky medical center; Tel-aviv; Israel

Keywords: RET gene; Colorectal cancer; Polyp; Adenoma; Chromosome; Transmembrane receptor tyrosine; Papillary; Medullary thyroid cancers; Multiple endocrine neoplasm

Abbrevations: MEN: Multiple Endocrine Neoplasm Type; CRC: Colorectal Cancer; CT: Cycle Threshold; PJS: Peutz-Jeghers Syndrome; GI: Gastrointestinal; HGD: High Grade Dysplasia; STAT: Signal Transducer and Activator of Transcription

\section{Introduction}

The RET proto-oncogene is located on chromosome 10q11.2 and encodes a transmembrane receptor tyrosine kinase that has three unique isoforms [1]. Four ligands can bind and activate RET, leading to the aberrant activity of several signaling pathways including PI3K/Akt and MAPK pathway [2]. The aberrant expression of RET may function as an oncogene in certain solid tumor malignancies including papillary, medullary thyroid cancers and the multiple endocrine neoplasm type 2 (MEN2) syndrome $[3,4]$.

In colorectal cancer RET has been identified as a tumor suppressor and as an oncogene. Studies have shown that the hypermethylation and mutational inactivation of RET, as well as RET fusions, promote colorectal cancer formation [5-7].

Somatic RET gene mutations and rearrangement in CRC and in advanced colon adenomas are reported, with a prevalence of $0.9-8.9 \%$ [8]. In vitro studies have shown increased activity of the RET fusion kinase that demonstrated stimulation of anchorage-dependent proliferation, migration and cell survival [8-10]. However, there is a lack of evidence on whether germline RET gene mutations are associated with an increased risk for the development of colorectal cancer (CRC) or colorectal adenomas. The aim of this study was to assess the frequency of germline RET gene mutations in a cohort of subjects at high-risk for colorectal cancer.

\section{Materials and Methods}

This was a retrospective case-control study. The study was held in a tertiary referral center at the Gastrointestinal depart ment, Tel-Aviv Sourasky Medical Center, Tel-Aviv, Israel, which is a tertiary referral center. The study was approved by local and national ethics comities.

Over a three-year period (01.2015-12.2017), 140 subjects at high-risk for colorectal cancer were referred for genetic evaluation through multi-gene panel testing. Study cohort included subjects with: personal history of early-onset CRC $(<50$ years old $)$, or personal history of CRC at any age plus colorectal adenomatous polyps, or personal history of CRC or advanced colorectal adenoma plus a family history of CRC in one or more first degree relative.

\section{Genetic Analysis}

Genomic DNA from all the 140 study subjects was extracted from whole blood using dedicated kit (5 PRIME Inc., Gaithersburg, MD, USA, Archive Pure TM) as instructed by the manufacturer's guidelines. The genetic analysis was performed in GGA lab, by testing the enire genomic sequence of 72 cancer-related genes. Specifically, the 72 genes were analyzed using the Fluidigm Bio Mark apparatus (Fluidigm Corp.) with assay-specific TaqMan fluorescence primer/probe mixes [11].

Assays along with PCR master mix were run in duplicate by loading 5 microliter into each well of the primed 96.96 Fluidigm Chip. The chip was then placed in the integrated fluidic circuit controller and loaded before analysis with the BioMark reader. The following thermal cycling protocol was used: 50uC $(2 \mathrm{minu}-$ tes), 70uC (30 minutes), 25uC (10 min), 50uC ( 2 minutes), and $95 \mathrm{uC}$ ( 4 minutes). This was followed by 40 cycles of $95 \mathrm{uC}(10$ 
seconds) and $61 \mathrm{uC}(30$ seconds, the final cycle [50uC (2 minutes), $70 \mathrm{uC}$ (30 minutes), 25uC (10 minutes)]. Data were analyzed and cycle threshold (CT) values were determined using BioMark real-time PCR analysis software (Fluidigm Corp.), and automated mutation calling was carried out using an algorithm based on the change in CT values between wild-type and mutant or between control and mutant, for TaqMan assays, respectively. All positive results (mutations detected) were confirmed by Sanger sequencing. Segregation analysis was performed through testing the probands' all available first-degree family members for the mutations detected.

\section{Results}

Out of the 140 subjects tested, seven (5\%) were identified to have a germline mutation in the RET gene. Table 1 shows RET gene mutation carriers clinical and genetic characteristics.

Table 1: RET gene mutation carriers.

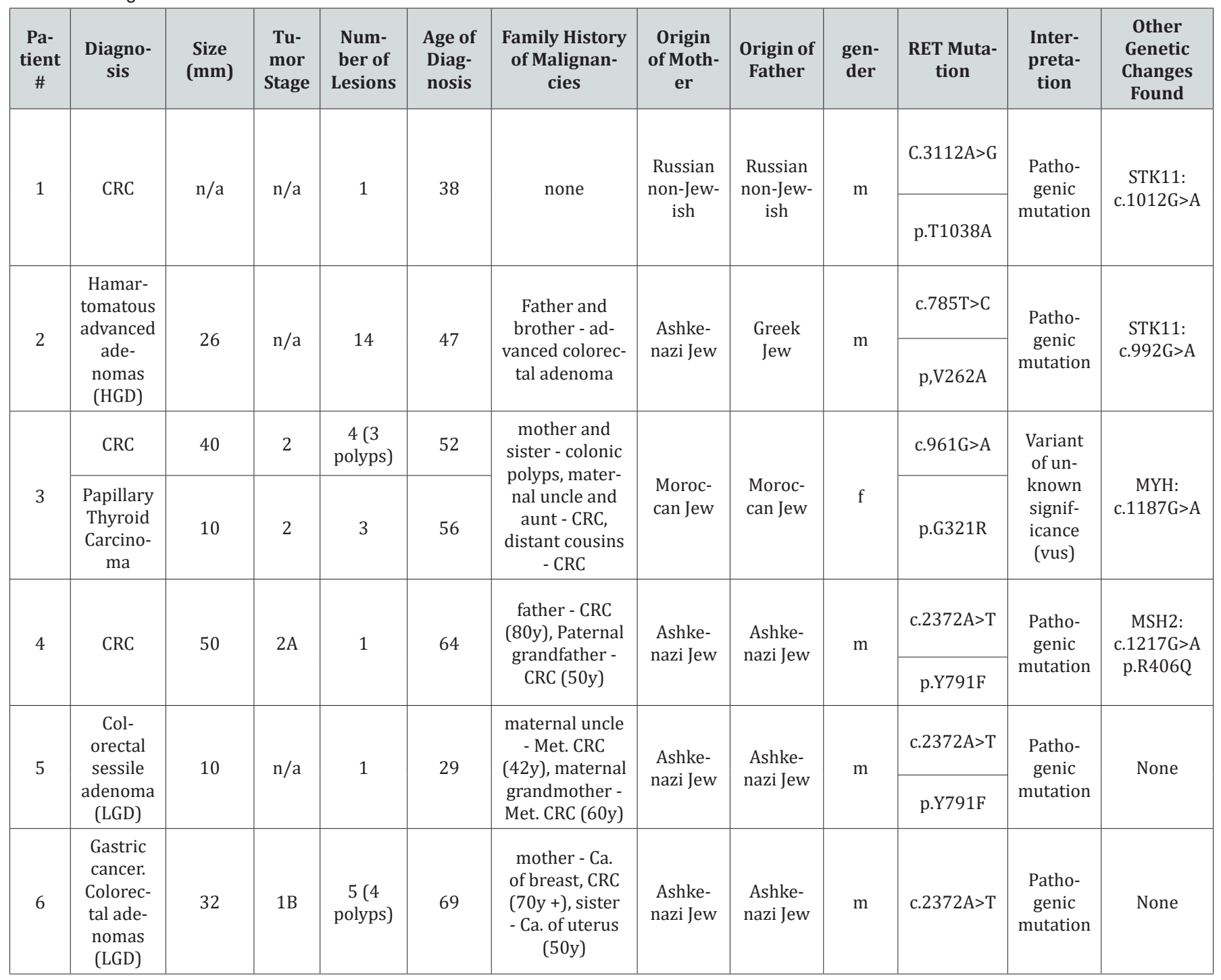

In five subjects (of the 7 RET mutation carriers), additional germline genetic changes were found, so that we cannot determine to what extent, these genetic changes contributed to the clinical picture (phenotype) in each case. However, the RET mutations detected here certainly had some effect on the carcinogenesis sequence occurred in our cases. In more detail (Table 1): out of the 7 subjects with RET mutations: two subjects had an additional genetic alteration, detected in the STK11 gene, the gene responsible for Peutz-Jeghers syndrome (PJS). Individuals affected by PJS have an increased occurrence of gastrointestinal (GI) hamartomas [12], and have an increased risk for cancer including breast, pancreas and gynecological.
Subject \#1 was found to carry the c.3112A>G RET mutation and did not have any of the classical PJS manifestations. He was diagnosed with CRC at the age of 38 and did not had any hamartomatous polyps during $>10$ years of colonoscopies follow-up. The additional genetic change detected in the STK11 gene: c. $1012 \mathrm{G}>\mathrm{A}$, is reported as a variant of uncertain significance by genetic and bioinformatics databases like ClinVar and mutation taster. Hence, this patient phenotype cannot be explained by the STK11 variant. However, the RET mutation detected was predicted by genetic and bioinformatics databases as a pathogenic mutation, therefore could be associated with CRC development. The frequency of the RET mutation: c.3112A $>\mathrm{G}$ (coordinate: 
chr10:43622095) is 3/1148 in database of 574 Ashkenazi Jews [13], and 2/10152 in gnomAD [14].

Subject \#2 was found to carry the c.785T>C RET mutation and clinically had colorectal hamartomatous polyps as well as adenomas with high grade dysplasia (HGD). The hamartomaous polyps are to be expected in PJS individuals. The additional genetic cahnge detected in the STK11 gene: c. $992 \mathrm{G}>\mathrm{A}$, is reported as a variant of uncertain significance by genetic and bioinformatics databases [15-17] In addition, segregation analysis of the STK11 alteration in his family did not show clinical genotype-phenotype match. Interestingly, the RET alteration in the family did segregate with phenotype of advanced colorectal polyps in his father and one of his brothers, suggesting a possible role for this RET alteration in colorectal adenoma-carcinoma sequence in this family.

The frequency of the RET mutation: c.785T $>C$ (coordinate: chr10:43600559) is 0/1148 in database of 574 Ashkenazi Jews [13], and 1/10152 in gnomAD [14].

Subject \#3 was found to carry the c.961G>A RET mutation had colorectal cancer as well as few adenomas at age 52 and was diagnosed with papillary thyroid carcinoma at age 56. She was found to carry bi-allelic MUTYH mutation which could explain her clinical picture. The RET mutation (c.961G>A) is reported in bioinformatic databases as having "conflicting interpretation of pathogenicity". We could not determine if this RET alteration added to the cancer risk in this individual and to what extent.

The frequency of the RET mutation: c. $961 \mathrm{G}>\mathrm{A}$ (coordinate: chr10:43601917) is 0/1148 in database of 574 Ashkenazi Jews [13], and 0/10152 in gnomAD [14].

Subject \#4 was found to carry the c.2372A>T RET mutation and was diagnosed with CRC at age 64 years. In addition to a pathogenic nutation in the RET gene he was found to carry a genetic alteration in the MSH2 gene known to be related to HNPCC-Lynch syndrome [18]. However, Immunohistochemistry staining of the tumor tissue showed normal staining of the MSH2 and MSH6 as well as MLH1 and PMS2 proteins with stable microsatellite instability testing (MSS), therefore not supporting the role of MSH2 mutation as the driver mutation of colorectal carcinogenesis in this case. The frequency of the RET mutation: c. $2372 \mathrm{~A}>\mathrm{T}$ (coordinate: chr10:43613908) is 13/1148 in database of 574 Ashkenazi Jews [13], and 164/10152 in gnomAD [14].

Subject \#7 was found to carry the c.3256G>A RET mutation and had advanced high-grade dysplasia colorectal adenoma at the age of 53 years. In addition, he was found to carry a genetic alteration in the $\mathrm{CDH} 1$ gene (c.808T $>\mathrm{G}$ ) that is predicted as a polymorphism by bioinformatic databases [15-17]. The frequency of the RET mutation: c.3256G>A (coordinate: chr10:43623628) is $0 / 1148$ in database of 574 Ashkenazi Jews [13] and 3/9850 in gnomAD [14].

Interestingly, 3 of the 7 RET gene carriers had the same missense mutation (c.2372A>T, p. Y791F), predicted to be a pathogenic mutation by bioinformatic databases [15-17]. These three subjects are all Ashkenazi Jews, implying it as a possible founder mutation, and as shown here its' frequency is $1.13 \%$ [13].

\section{Discussion}

In this retrospective study RET gene mutations were found in $5 \%$ of cohort of subjects at high-risk for colorectal cancer. This finding was unexpected as to the best of our knowledge germline RET mutations were not considered to be associated with increased colorectal cancer risk. Interestingly, 3 of the 7 RET gene carriers had the same missense pathogenic mutation [15-17].

Menacho et al. [19] have showed that this specific mutation (p. Y791F) causing the RET protein to adapt a capability of a monomeric receptor, which is auto phosphorylated and activated independently of glial cell line-derived neurotrophic factor. The dysfunctional signaling properties of this mutant, when compared with wild-type RET, involve constitutive activation of signal transducer and activator of transcription 3 (STAT3). Three-dimensional modeling of the RET catalytic domain suggested that the structural changes promoted by the respective amino acid's substitutions lead to a more accessible substrate and ATP-binding monomeric conformation. They further showed that immunohistochemical analysis of FMTC tumor samples support the in vitro data, because of high degree of RET expression at the plasma membrane was observed. The three subjects from our cohort who carry this mutation are all Ashkenazi Jews, implying it as a possible founder mutation in Ashkenazi Jews, with a frequency of $1.13 \%$ based on genetic data in Ashkenazi Jews [13]

Six of the RET alterations found in our cohort are predicted as pathogenic mutations according to various bioinformatic databases [15-17]. Additional genetic changes other than in the RET gene were identified in four of the patients but as was mentioned in the results section, were unlikely to be responsible for the whole clinical picture in each case. There is a lack of evidence regarding germline RET gene mutations and CRC or colorectal adenomas. Current literature suggests an involvement of somatic RET gene mutations and rearrangement in CRC and in advanced colon adenomas, with a prevalence similar to what was found in our cohort $(3,4 \%)$ [8]. This finding strengthens the already known fact of RET protein and its downstream biological pathway as having an important role in at least part of the colorectal cancer cases (the SRC ? RAC10JNK pathway and the Ras?Raf? MEK?ERK pathways) [20].

Interestingly, none of the carriers had MTC, nor a family history of MTC. Possible explanation for this observation could be the affected downstream biological pathway involved by these mutations or the impact of the mutation on the RET gene, whether it affects its proto-oncogene capability or its tumor suppressor capability. The identification of CRC subjects carrying RET gene mutations raises the possibility for treatment options. Regorafenib (BAY 73-4506; Bayer Schering Pharma AG, Berlin, Germany) is an oral small-molecule multikinase inhibitor that is active against several angiogenic RTKs (VEGFR-1, VEGFR-2, VEGFR-3, TIE-2), oncogenic RTKs (c-KIT, RET), stromal RTKs (PDG- 
FR-B, FGFR1), and intracellular signaling kinases (c-RAF/RAF-1, BRAF, BRAFV600E) [20]. Several clinical studies performed on regorafenib showed positive results: The CORRECT trial demonstrated that regorafenib significantly increased overall survival, progression free survival versus placebo in patients with chemo refractory metastatic CRC [21]. Based on the results of this study, regorafenib was approved by the US FDA in September 2012 for the treatment of patients with refractory metastatic CRC. Another study showed that there is an in-vitro cytotoxic effect on RET-kinase fusion protein positive CRC cells exposed to regorafenib and that rapid decrease in CEA and LDH in affected subjects can serve as bio-markers for favorable clinical response [7].

\section{Conclusion}

In conclusion, in this study we found RET gene mutations in $5 \%$ of subjects at high-risk for colorectal cancer. This finding suggest that RET mutations have a role in increasing colorectal cancer risk. Further studies are needed to validate these results and to investigate the role of thyroid kinase inhibitors as a possible treatment or preventive option.

\section{References}

1. Runeberg-Roos P, Saarma M (2007) Neurotrophic factor receptor RET: structure, cell biology, and inherited diseases. Ann Med 39(8): 572 580

2. Arighi E, Borrello MG, Sariola H (2005) RET tyrosine kinase signaling in development and cancer. Cytokine Growth Factor Rev 16(4-5): 441467

3. Ahn DH, Ciombor KK, Mikhail S, Bekaii-Saab (2016) Genomic diversity of colorectal cancer: Changing landscape and emerging targets. World J Gastroenterol 22(25): 5668-5677.

4. Phay JE, Shah MH (2010) Targeting RET receptor tyrosine kinase activation in cancer. Clin Cancer Res 16(24): 5936-5941.

5. Luo Y, Tsuchiya KD, Park D, Fausel R, Kanngurn S, et al. (2013) RET is a potential tumor suppressor gene in colorectal cancer. Oncogene 32(16): 2037-2047

6. Mokarram P, Kumar K, Brim H, Naghibalhossaini F, Saberi-firoozi M, et al. (2009) Distinct high-profile methylated genes in colorectal cancer. PLoS One 4(9): e7012.

7. Le Rolle AF, Klempner SJ, Garrett CR, Seery T, Sanford EM, et al. (2015) Identification and characterization of RET fusions in advanced colorectal cancer. Oncotarget 6(30): 28929-28937.

8. Lee SH, Jung SH, Kim TM, Rhee JK, Park HC, et al. (2017) Wholeexome sequencing identified mutational profiles of high-grade colon adenomas. Oncotarget 8(4): 6579-6588.
9. Hechtman JF, Zehir A, Yaeger R, Wang L, Middha S, et al. (2016) Identification of Targetable Kinase Alterations in Patients with Colorectal Carcinoma That are Preferentially Associated with WildType RAS/RAF. Mol Cancer Res 14(3): 296-301.

10. Mendes Oliveira D, Grillone K, Mignogna C, De Falco V, Laudanna C, et al. (2018) Next-generation sequencing analysis of receptor-type tyrosine kinase genes in surgically resected colon cancer: identification of gainof-function mutations in the RET proto-oncogene. J Exp Clin Cancer Res 37(1): 84 .

11. Wang J, Lin M, Crenshaw A, Hutchinson A, Hicks B, et al. (2009) High-throughput single nucleotide polymorphism genotyping using nanofluidic Dynamic Arrays. BMC Genomics 10: 561.

12. Beggs AD, Latchford AR, Vasen HF, Moslein G, Alonso A, et al. (2010) Peutz-Jeghers syndrome: a systematic review and recommendations for management. Gut 59(7): 975-986.

13. Lencz T, Yu J, Palmer C, Carmi S, Ben-Avraham D, et al. (2018) Highdepth whole genome sequencing of an Ashkenazi Jewish reference panel: enhancing sensitivity, accuracy, and imputation. Hum Genet 137(4): 343-355.

14. Lek M, Karczewski KJ, Minikel EV, Samocha KE, Banks E, et al. (2016) Analysis of protein-coding genetic variation in 60,706 humans. Nature 536(7616): 285-291.

15. Fokkema IF, Taschner PE, Schaafsma GC, Celli J, Laros JF, et al. (2011) LOVD v.2.0: the next generation in gene variant databases. Hum Mutat 32(5): 557-563.

16. Landrum MJ, Lee JM, Benson M, Brown G, Chao C, et al. (2016) ClinVar: public archive of interpretations of clinically relevant variants. Nucleic Acids Res 44: D862-868.

17. Schwarz JM, Cooper DN, Schuelke M, Seelow D, et al. (2014) Mutation Taster2: mutation prediction for the deep-sequencing age. Nat Methods 11(4): 361-362.

18. Carethers JM, Stoffel EM (2015) Lynch syndrome and Lynch syndrome mimics: The growing complex landscape of hereditary colon cancer. World J Gastroenterol 21(31): 9253-9261.

19. Plaza Menacho I, Koster R, Van der Sloot AM, Quax WJ, Osinga J, et al. (2005) RET-familial medullary thyroid carcinoma mutants Y791F and S891A activate a Src/JAK/STAT3 pathway, independent of glial cell line-derived neurotrophic factor. Cancer Res 65(5): 1729-1737.

20. Goel G (2018) Evolution of regorafenib from bench to bedside in colorectal cancer: Is it an attractive option or merely a "me too" drug? Cancer Manag Res 10: 425-437.

21. Grothey A, Van Cutsem E, Sobrero A, Siena S, Falcone A, et al. (2013) Regorafenib monotherapy for previously treated metastatic colorectal cancer (CORRECT): an international, multicentre, randomised, placebo-controlled, phase 3 trial. Lancet 381(9863): 303-312. 
